inability on part of the patient to bear the expenses. The management of condylar fractures has been a topic of controversy since ages, with advocates as well as critics of both the conservative and invasive techniques. ${ }^{[3]}$ The conservative option ranges from no treatment, to variable period of IMF, using different types of wiring techniques such as: wired arch bar, eyelets, cap splint, interdental wiring, stout wiring, bracket bar, Leonard button, otten mini hooks and ulster hook. The most commonly used IMF technique for management of facial trauma is the wired arch bar. Although it provides sufficient immobilisation, it has a number of disadvantages: it is a cumbersome procedure, there is an increased risk of needle stick injury (because of increased number of wires used) and maintaining gingival health is difficult. ${ }^{[4]}$ Keeping these facts in mind, we attempted to design a simple wiring technique that can provide sufficient immobilisation as well as avoid the above-mentioned drawbacks.

Method of interdental wire hook fabrication: A 26-gauge, $10 \mathrm{~cm}$ long wire was used in the current technique. We applied a mark at $3 \mathrm{~mm}$ distance from one end of the wire. This end was turned to form a loop with the remaining wire and then another turn was given in the same direction holding the previously formed loop. The whole wire was passed through the first loop to form a hook. This hook was kept at right angle to the wire during the IMF. Wire hooks were pre-fabricated before starting the procedure and eight of them were applied interdentally [Figure $1 \mathrm{a}$ and $\mathrm{b}$ ].

\title{
A novel wiring technique in mandibular condylar fracture: customised interdental wire hooks
}

Sir,

Intermaxillary fixation (IMF) is the oldest and most widely used technique of jaw immobilisation for management of facial trauma. Immobilisation of the fractured bone segments aims to reduce the motion along the continuity disruption, which is important to promote timely healing or union. ${ }^{[1]}$ Although technology has advanced to a position where every maxillofacial fracture can be treated by open reduction and internal fixation (ORIF) with ease, ${ }^{[2]}$ traditional conservative methods still hold their place in management of facial fractures in some situations like unavailability of required armamentarium for ORIF or
Method of IMF technique: After aseptic preparation, 2\% lignocaine jelly was applied for topical anaesthesia if required. Wire hooks were placed interdentally on one side of maxilla and mandible as follow:

1. Between first and second molars in maxilla (from buccal to palatal and wire end from palatal to buccal projected between the second premolar and first molar).

2. Between premolar and first molar in mandible (from buccal to lingual and wire end from lingual to buccal projected between first and second molar).

3. Between premolar and canine (wire end projected between premolars) and between premolars (wire end projected between canine and premolar) in maxilla and mandible, respectively.

4. Reverse order was used on opposite side in maxilla and mandible.

5. After placing all wire hooks, tightening of upper wire ends was done with respective lower wire ends [Figures 2 and 3]. 


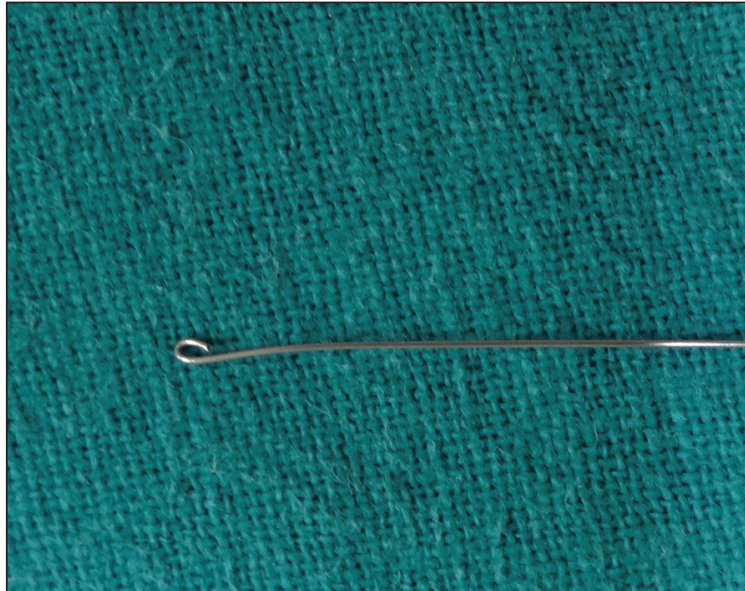

Figure 1a: Interdental wire hook fabrication ( $1^{\text {st }}$ band $)$

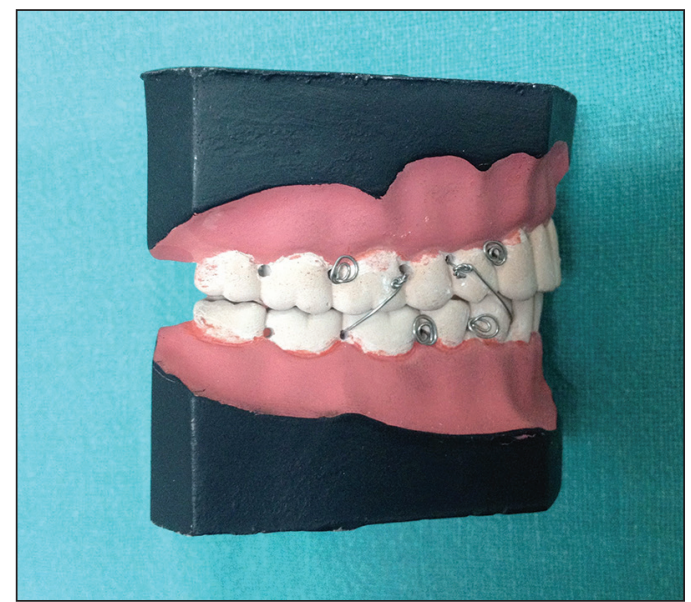

Figure 2: Study model demonstrating the intermaxillary fixation using interdental wire hook

According to the authors, the ideal IMF technique should have the following features:

1. It should be simple and quick.

2. It should have minimal number of wires in the oral cavity.

3. It should allow maintenance of good oral hygiene and gingival health during IMF period.

4. If possible, it should require minimal or no local/ topical anaesthesia.

5. It should provide adequate immobilisation for particular fracture stability.

Wired arch bar although is the gold standard among IMF techniques, but it requires long operating time. It is a cumbersome procedure where a large number of wires are required, which raises the risk of needle stick injury to the operating surgeon..$^{[4]}$ Moreover, at times, there are fractures like minimal to moderately displaced mandibular condylar fractures, which require a lesser degree of immobilisation and for only short duration.

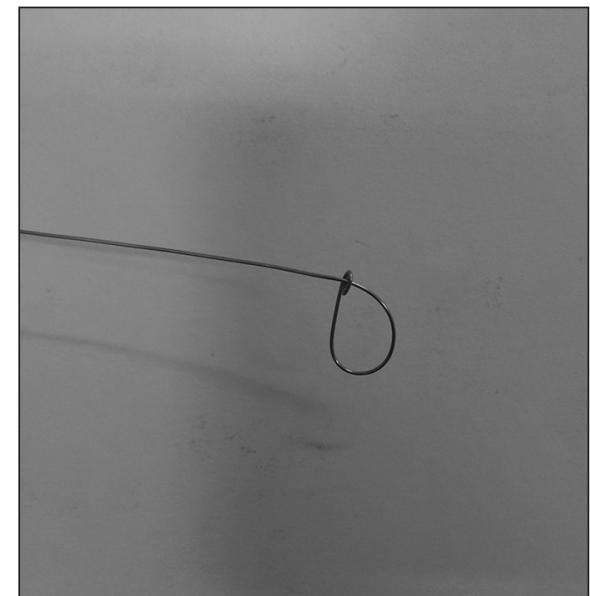

Figure 1b: Interdental wire hook fabrication (final band)

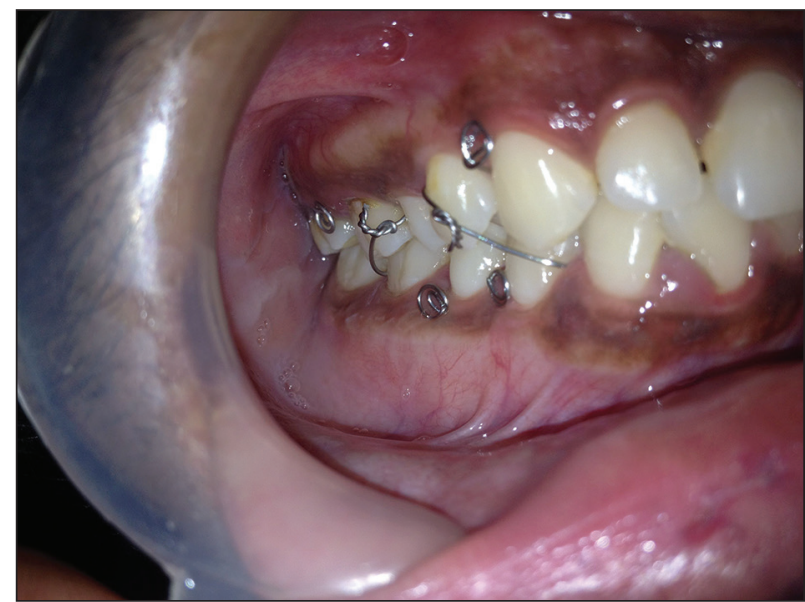

Figure 3: Clinical photograph with intermaxillary fixation using interdental wire hook

In this type of condition, the authors feel that the bulky wired arch bars can be substituted with another technique having less bulk of wires, which is less tedious, quick and simple.

The new wiring technique has all the above-mentioned features. However, for obtaining good results, proper case selection is of paramount importance. The authors have used this wiring technique in condylar and non-displaced dentate mandibular fractures as a mean of immobilisation. The wiring technique that is described here by no means is meant to surpass the already described techniques. However, authors advocate this technique as a mean of immobilisation for fractures requiring a lesser degree of immobilisation for shorter duration.

\section{Amita Rani, Jitender Dabas, Sujata Mohanty}

Department of Oral and Maxillofacial Surgery, Maulana Azad Institute of Dental Sciences, New Delhi, India

Indian Journal of Plastic Surgery January-April 2015 Vol 48 Issue 1 
Address for correspondence:

Dr. Amita Rani, Department of Oral and Maxillofacial Surgery, Maulana Azad Institute of Dental Sciences, BSZ Marg,

Near ITO, New Delhi - 110 002, India.

E mail: poswal.dramita152@gmail.com

\section{REFERENCES}

1. McGinn JD, Fedok FG. Techniques of maxillary - Mandibular fixation. Oper Tech Otolaryngol 2008;19:117-22.

2. Kamath RA, Bharani S, Hammannavar R, Ingle SP, Shah AG. Maxillofacial trauma in central Karnataka, India: An outcome of 95 cases in a regional trauma care centre. Cranial Maxillofac Trauma Reconstr 2012;05:197-204.

3. Joos $U$, Kleinheinz J. Therapy of condylar neck fractures. Int J Oral Maxillofac Surg 1998;27:247-54.

4. Carlton JE, Dodson TB, Cleveland JL, Lockwood SA. Percutaneous injuries during oral and maxillofacial surgery procedures. J Oral Maxillofac Surg 1997;55:553-6.

\begin{tabular}{|l|l|}
\hline \multicolumn{3}{|c|}{ Access this article online } \\
\hline Quick Response Code: & Website: \\
\hline & www.ijps.org \\
\hline & \\
\hline
\end{tabular}

